\title{
Edge and waveguide terahertz surface plasmon modes in graphene microribbons
}

\author{
A. Yu. Nikitin,,${ }^{1,2}{ }^{*}$ F. Guinea ${ }^{3}$ F. J. García-Vidal, ${ }^{4}$ and L. Martín-Moreno ${ }^{1, \dagger}$ \\ ${ }^{1}$ Instituto de Ciencia de Materiales de Aragón and Departamento de Física de la Materia Condensada, CSIC-Universidad de Zaragoza, \\ E-50009, Zaragoza, Spain \\ ${ }^{2}$ A. Ya. Usikov Institute for Radiophysics and Electronics, Ukrainian Academy of Sciences, 12 Academician Proskura Street, \\ 61085 Kharkov, Ukraine \\ ${ }^{3}$ Instituto de Ciencia de Materiales de Madrid, CSIC, Cantoblanco, E-28049 Madrid, Spain \\ ${ }^{4}$ Departamento de Física Teórica de la Materia Condensada, Universidad Autónoma de Madrid, E-28049 Madrid, Spain
}

(Received 28 July 2011; revised manuscript received 22 September 2011; published 24 October 2011)

\begin{abstract}
Surface plasmon modes supported by graphene ribbon waveguides are studied and classified. The properties of both modes with the field concentration within the ribbon area (waveguiding modes) and on the edges (edge modes) are discussed. The waveguide and edge modes are shown to be separated from each other by a gap in wave numbers. The even-parity hybridized edge mode results to be the fundamental electromagnetic mode of the ribbon, possessing also the lowest losses. All of the plasmonic modes in the ribbons have an optimum frequency, at which the absorption losses are minimum, due to compromise between the plasmon confinement and the decrease of the group velocity close to the modal cutoff. The presented results show that the diffraction limit in graphene ribbons can be surpassed.
\end{abstract}

DOI: 10.1103/PhysRevB.84.161407

PACS number(s): 78.66.Bz, 42.25.Bs, 41.20.Jb, 42.79.Ag

The science of graphene is advancing rapidly, discovering unique fundamental physical effects ${ }^{1}$ and showing promising perspectives in several state-of-the-art technological applications. ${ }^{2}$ For example, graphene can carry both electromagnetic (EM) signals and electric currents through the same extremely thin circuitry, which could allow electrical interconnects to achieve data transmission faster rates. In this regard, it has been recently shown that graphene ribbons can operate as a broadband radio-frequency mixer at frequencies up to $10 \mathrm{GHz}$ inside an integrated circuit, ${ }^{3}$ showing a great potential for waveguiding. The two main characteristics of graphene for this kind of application are (i) that, despite being almost transparent, graphene can support surface plasmons (GSPs) in the $\mathrm{THz}$ regime ${ }^{4,5}$ and (ii) the carrier concentration (and thus the conductivity) in graphene can be controlled through electrostatic gating. This last property can, in turn, be used to tune the properties of GSPs, opening up a wealth of interesting applications in photonics. ${ }^{2,6-12}$

For most functionalities the GSPs must ideally also be confined laterally in the graphene sheet, which is known to occur in graphene ribbons. ${ }^{6,9,10,13,14}$ However, a characterization of the confined plasmons in this geometry is lacking. In this Rapid Communication we address this problem, and study the characteristics of GSP modes in graphene ribbons of micrometric widths, in the $\mathrm{THz}$ regime. We concentrate on the spectral regimes where the ribbon supports either a single EM mode or a few of them and, in this last case, on the properties of strongly localized edge GSP modes (EGSP) that appear in the system. The dependence of both waveguide GSP (WGSP) and EGSP upon the relaxation time of electrons, permittivity of a substrate, and width of the ribbon is also analyzed.

Let us consider a graphene ribbon of width $w$ (at $|x|<$ $w / 2$ ), placed at the boundary $z=0$ between two dielectric half spaces (see Fig. 1). The (frequency-dependent) twodimensional conductivity of graphene is $\sigma(\omega)$. The ribbon can be either an actual graphene strip, or "virtually" created by spatially varying external gates acting on a graphene sheet, as

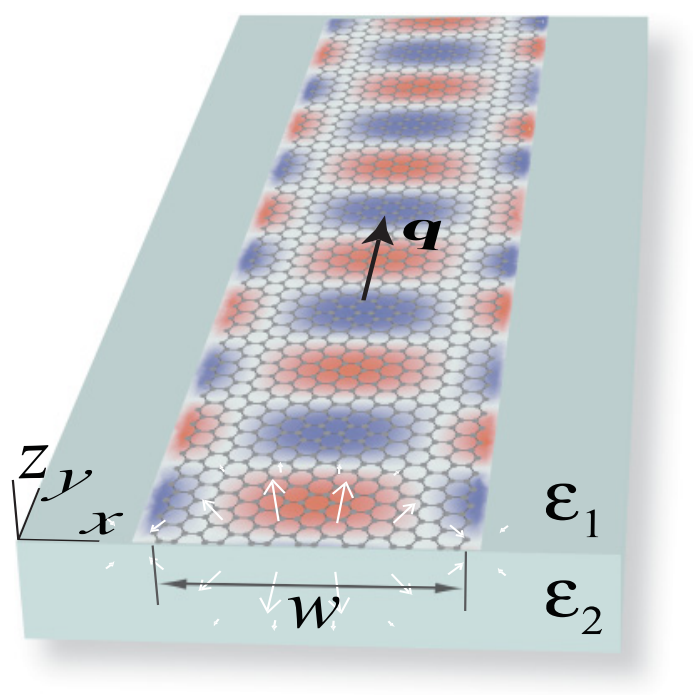

FIG. 1. (Color online) The geometry of the studied system: A graphene ribbon of the width $w$, and conductivity $\sigma$, placed at the interface between two dielectric half spaces with dielectric constants $\varepsilon_{1}$ and $\varepsilon_{2}$. The mode propagates along the $O y$ axis. The color plot presents an example of the electric field $x$-component spatial distribution in the $X O Y$ plane, and the arrows correspond to the electric field lines in the $X O Z$ plane.

proposed in Ref. 10. Here we only report on the case where $\sigma=0$ outside the ribbon, but we have checked that our results are virtually unmodified if this constrain is relaxed, provided GSPs are not supported by graphene for $|x|>w / 2$.

Due to translational symmetry, the electric field of each EM eigenmode in the ribbon has the form $\vec{E}(\vec{r}, t)=$ $\vec{E}(x, z) \exp \left(i q k_{\omega} y\right) \exp (-i \omega t)$, where $k_{\omega}=\omega / c$ is the freespace wave vector, $c$ is the speed of light, and $q(\omega)$ is the modal wave vector in dimensionless units. We will refer to the real part of $q(\omega)$ as the "normalized wave vector," while its imaginary part provides the propagation length of GSPs, 
$L$, through $L / \lambda=1 /[2 \pi \operatorname{Im}(q)]$, where $\lambda=2 \pi / k_{\omega}$ is the wavelength in vacuum.

For the calculations, we use the conductivity computed within the standard random-phase approximation, ${ }^{15-17}$ which depends on temperature $(T)$, chemical potential $(\mu)$, and scattering time $(\tau)$. Notice that for relatively small frequencies $\hbar \omega / \mu \ll 1$ (where the intraband contribution to the conductivity dominates) the real part of the conductivity is sensitive to $\tau$, while for higher frequencies $\hbar \omega / \mu \gtrsim 1$ (with a dominating interband contribution) the losses are predominantly temperature dependent. We chose $T=300 \mathrm{~K}, \mu=0.2 \mathrm{eV}$ and, unless otherwise stated, a relaxation energy $E_{\tau}=2 \pi \hbar / \tau=0.1 \mathrm{meV}$ (corresponding to a mobility of $1.87 \times 10^{6} \mathrm{~cm}^{2} \mathrm{~V}^{-1} \mathrm{~s}^{-1}$ ). This value is chosen from the theoretical estimation of the maximum mobility in graphene. ${ }^{18}$ The present experimental values are $\sim 0.1-0.5$ times smaller, but the reported record value keeps increasing. All calculations have been performed by using the finite-element commercial software COMSOL.

In Fig. 2 both the dispersion relations and the propagation lengths of GSPs are rendered, for two widths of freestanding graphene ribbons. These widths have been chosen so that, in the considered frequency range, $w / \lambda<1$ for the 5 - $\mu$ m-width waveguide and $w / \lambda \lesssim 1$ for the $20-\mu \mathrm{m}$-width one. Notice that the dispersion relation is given in the form $q(v)$ with $v=2 \pi \omega$, so that $q=1$ sets the position of the light cone. The normalized wave vectors are shown by continuous curves, while the propagation lengths are represented by discontinuous ones.

We would like to notice that the microscopic structure of graphene edges modifies significantly the electronic spectrum, especially at energies near the Dirac point. The calculations reported here are not affected by the microscopic details of the edges. We consider graphene stripes where the separation between the Fermi energy and the Dirac point is much larger than other scales, such as temperature or relaxation energy. At these energies, the main differences between different types of edges is the amount of intervalley scattering that they induce, which vanishes for a zigzag edge and is maximum for armchair edges. The macroscopic analysis discussed here can be written in terms of contributions from the two valleys. However, plasmons are collective excitations of the total electronic charge. An electric current from a given valley incident at a zigzag edge is reflected in the same valley, while an armchair edge leads to a change of valley upon reflection. The total current is, obviously, conserved in both cases. As the calculation depends only on total charges and total currents, the nature of the edge cannot change the results. An influence of the edge structure can be expected, however, near the Dirac point, where the combination of quantum confinement and microscopic structure leads to changes in the electronic spectrum.

The dispersion relation for GSP modes in ribbons can be related to that for (i) 2DGSP modes in an infinite graphene sheet (with wave vector $q^{2 \mathrm{D}}=\sqrt{1-1 / \alpha^{2}}$, where $\alpha=2 \pi \sigma / c$; see curve "2DGSP" in Fig. 2) and (ii) surface plasmon modes appearing at the edges in a semiinfinite graphene sheet ${ }^{10}$ (EGSPs) with wave vector $q^{E}(v)$ (curve "EGSP" in Fig. 2). Notice that the dispersion curve for a EGSP lies above that for a 2DGSP, which implies that the former is more tightly confined.

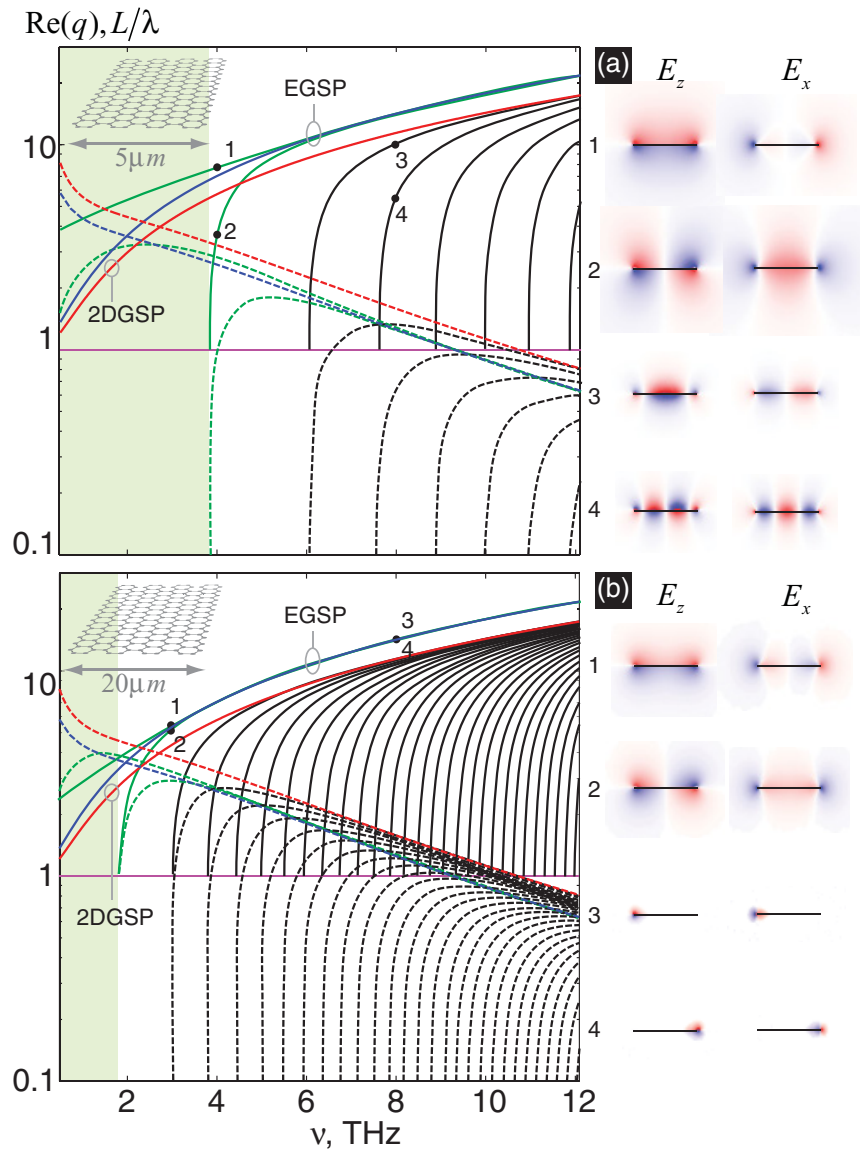

FIG. 2. (Color online) Normalized wave vector of GSP modes (continuous curves) and propagation lengths (discontinuous curves) as a function of frequency for freestanding graphene ribbons of $5-\mu \mathrm{m}$ (a) and $20-\mu \mathrm{m}$ (b) widths. The curve for dispersion law "EGSP" is for edge GSPs in a semi-infinite graphene sheet, while the one marked as "2DGSP" is for GSP in an infinite graphene sheet. The color plots for the $x$ and $z$ components of the electric field are shown in the vicinity of the ribbon cross section. The numbers next to the color plots correspond to the labels on the dispersion curves.

The presence of edges not only modifies the dispersion branches of GSPs, but also their polarization. The electric field gains an $x$ component of the field, i.e. in-plane component perpendicular to the propagation direction. This component is especially pronounced in the vicinity of the edges, where the field is maximally distorted compared to the case of 2DGSPs (see an example of the field distribution in Fig. 1).

We find that in a ribbon there are two modes that originate from the hybridization of EGSPs. Due to splitting, one of them (the one corresponding to even parity of $E_{z}$ with respect to the ribbon axis), has a wave vector that is larger than $q^{E}$ and is the fundamental one in the ribbon, while the other one has $q<q^{E}$. As seen from this figure, for both cases there is a single-mode region (shaded) for low frequencies. The wave vector of the fundamental mode is larger than that of 2DGSPs, and its propagation length is smaller, so $L$ remains of the order of 10 GSP wavelengths throughout the considered frequency range. For sufficiently large frequencies $(v=3.8 \mathrm{THz}$ for $w=$ $5 \mu \mathrm{m}$ and $v=1.8 \mathrm{THz}$ for $w=20 \mu \mathrm{m}$ ) a second GSP mode is sustained by the ribbon. As the frequency increases, the 


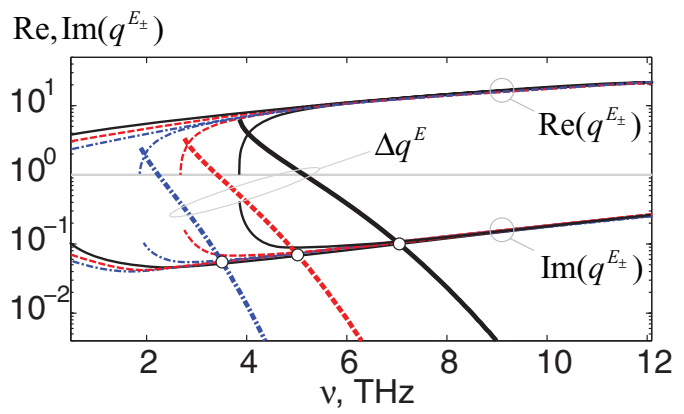

FIG. 3. (Color online) Difference between the normalized wave vectors of the two coupled EGSP ribbon modes $\Delta q$, together with $\operatorname{Re}\left(q^{E_{ \pm}}\right)$and $\operatorname{Im}\left(q^{E_{ \pm}}\right)$(corresponding to the edge EM modes of a semi-infinite graphene sheet). The continuous, dashed, and dasheddotted curves correspond to $w=5,10$, and $20 \mu \mathrm{m}$, respectively. The white circles indicate the points where $\operatorname{Im}\left(q^{E}\right)=\Delta q$, when the modes can be considered as degenerated.

dispersion relations for these two GSP modes approach each other, merging into the dispersion relation for an EGSP of a semi-infinite sheet. For a given frequency, the smaller is the width of the ribbon, the more the edge modes overlap and, correspondingly, the larger is the splitting between their wave vectors $q^{E+}$ and $q^{E-}$. The highest frequency for which the two edge modes can be considered as coupled can be estimated from the relation $\Delta q^{E}<\max \left\{\operatorname{Im}\left(q^{E_{ \pm}}\right)\right\} \simeq \operatorname{Im}\left(q^{E}\right)$, which means that the "linewidth" (due to absorption) of the isolated EM edge mode prevails the splitting (see Fig. 3).

Additionally to these hybridized edge modes, the ribbon may support several WGSPs having a field that extends over the ribbon width. The number of WGSPs trapped by the ribbon can be estimated as $N_{w} \sim 2 q^{2 \mathrm{D}} w / \lambda$, which counts basically how many GSP half wavelengths fit across the ribbon. These "bulk" modes present a dependence for $q(v)$ that lies below the corresponding one for a 2DGSP, which is their high-frequency asymptote (see Fig. 2). Nevertheless, the propagation length of these bulk modes is always smaller that the one for the 2DGSPs (see Fig. 2).

As seen from Fig. 2, for all EM modes in the graphene ribbon, the propagation length decays strongly close to the modal cutoff, which is much smaller than the one in a two-dimensional (2D) graphene sheet, and coincides with the latter for high frequencies. Between these two regions, each curve for $L / \lambda$ has a maximum at a finite frequency. To understand such peculiar behavior, let us first turn to a very interesting property of 2DGSPs. In the studied frequency range the real part of the conductivity (responsible for the dissipation) decreases as the frequency increases. However, due to increase of $\operatorname{Re}(q)$, the confinement increases so quickly with the frequency increase that GSPs become more absorptive, and consequently the propagation length of 2DGSPs decreases. Returning to the modes in the ribbons, in the region of high frequencies, where their $\operatorname{Re}(q)$ are large, the confinement of the modes is high and their absorption increases with the frequency, exactly as in the case of 2DGSPs. For the frequencies close to the modal cutoff, where $\operatorname{Re}(q)$ is not as large, the group velocity strongly decreases and losses get accumulated over a very short distance. Thus, the maxima in the propagation lengths correspond to the compromise between strong plasmon confinement (high frequencies) and low group velocities (lower frequencies).

We now consider how the WGSP characteristics depend on different parameters. Their dependence on the relaxation energy is represented in Fig. 4(a), showing that, while the propagation length of the modes is very sensitive to $E_{\tau}$ in this frequency regime, their wave vector is practically unaffected by it. This is related to the fact that the imaginary part of the conductivity is almost independent upon $\tau$, while the real part strongly depends on it, especially for lower frequencies. With a frequency increase, the GSPs losses become less effected by the relaxation time of electrons being more sensitive to the temperature.

With respect to the presence of a substrate, our calculations show that the dispersion relation of WGSP has the same structure as that shown in Fig. 2 for the freestanding case. Figure 4(b) renders, as a function of the dielectric constant of the substrate $\left(\varepsilon_{2}\right)$, the spectral value of the "asymptotes," i.e., the wave vector and propagation length for both the EGSP supported by a semi-infinite graphene sheet (discontinuous lines) and the 2DGSP (continuous lines). As $\varepsilon_{2}$ increases, the GSPs become more localized $[\operatorname{Re}(q)$ increases and, correspondingly, the propagation length decreases]. In the
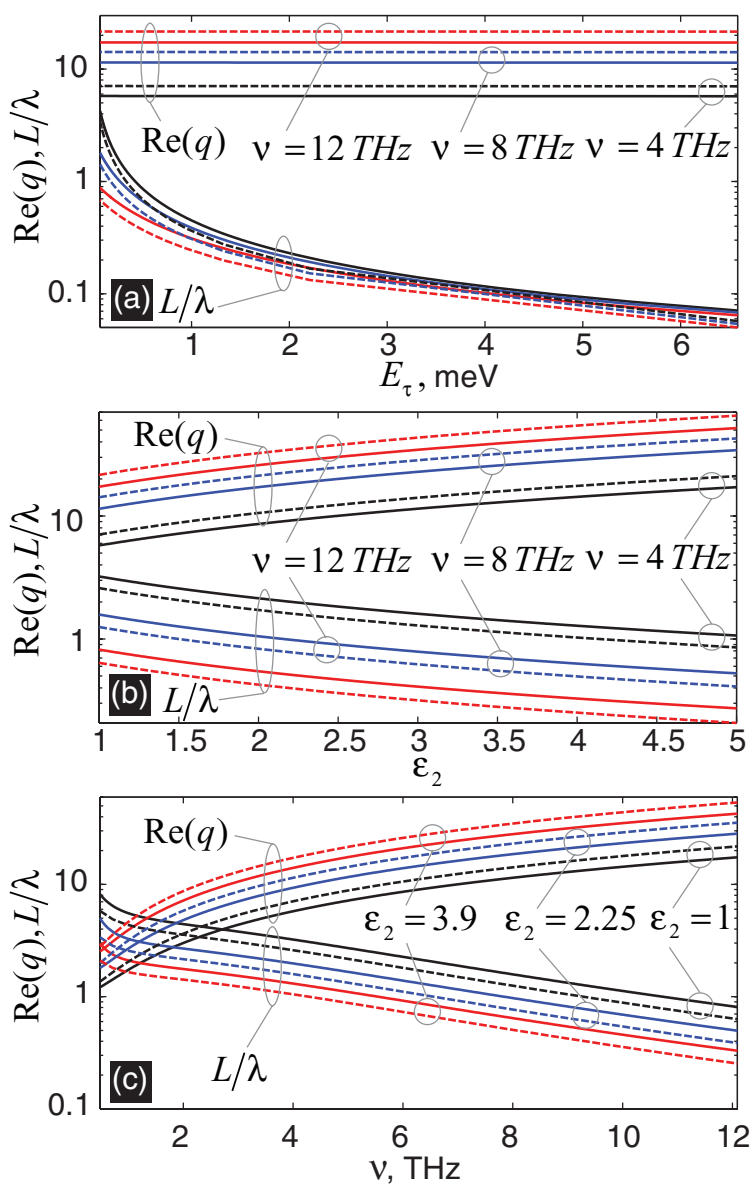

FIG. 4. (Color online) Dependencies of $\operatorname{Re}(q)$ and $L$ (discontinuous lines: EGSP of a semi-infinite sheet; continuous lines: 2DGSP) with (a) scattering time (in units of $\mathrm{meV}$ ), (b) dielectric constant of the substrate, and (c) frequency. Unless otherwise stated, $\varepsilon_{2}=1$ and $E_{\tau}=0.1 \mathrm{meV}$. 
region of high frequencies, where the momentum is very large, $q \gg 1$, the GSPs are less sensitive to the dielectric constant of the substrate.

To conclude, we have studied the dispersion characteristics of SP modes existing in graphene ribbons in the $\mathrm{THz}$ frequency range. We have shown that there are two types of SP modes: the waveguide type, with the field concentrated along the whole area of the ribbon (in the $x$ direction), and the edge modes, with the field concentrated on the rims of the ribbons, $x= \pm w / 2$. The waveguide and edge modes are separated by a wave-vector gap from each other. The number of SP modes supported by the ribbon increases as either the frequency or the ribbon width increases. The wave vector of the modes is not sensitive to the relaxation time of charge carriers, but the propagation length is strongly affected. The SP modes can be tuned by changing the dielectric environment of the ribbon. The high localization of graphene EM edge modes can be useful for, for instance, bending of EM signals on subwavelength scales and enhancing the EM coupling between objects.

The authors acknowledge support from the Spanish MECD under Contract No. MAT2009-06609-C02 and Consolider Project "Nanolight.es." A.Y.N. acknowledges the Juan de la Cierva Grant No. JCI-2008-3123. *alexeynik@rambler.ru

${ }^{\dagger} 1 \mathrm{~mm} @$ unizar.es

${ }^{1}$ A. H. Castro Neto, F. Guinea, N. M. R. Peres, K. S. Novoselov, and A. K. Geim, Rev. Mod. Phys. 81, 109 (2009).

${ }^{2}$ F. Bonaccorso, Z. Sun, T. Hasan, and A. C. Ferrari, Nat. Photon. 4, 611 (2010).

${ }^{3}$ Yu-M. Lin, A. Valdes-García, S.-J. Han, D. B. Farmer, I. Meric, Y. Sun, Y. Wu, C. Dimitrakopoulos, A. Grill, P. Avouris, and K. A. Jenkins, Science 332, 1294 (2011).

${ }^{4}$ M. Jablan, H. Buljan, and M. Soljačić, Phys. Rev. B 80, 245435 (2009).

${ }^{5}$ A. A. Dubinov, V. Ya. Aleshkin, V. Mitin, T. Otsuji, and V. Ryzhii, J. Phys. Condens. Matter 23, 145302 (2011).

${ }^{6}$ V. V. Popov, T. Yu. Bagaeva, T. Otsuji, and V. Ryzhii, Phys. Rev. B 81, 073404 (2010).

${ }^{7}$ G. W. Hanson, J. Appl. Phys. 103, 064302 (2008).
${ }^{8}$ Yu. V. Bludov, M. I. Vasilevskiy, and N. M. R. Peres, Europhys. Lett. 92, 68001 (2010).

${ }^{9}$ E. G. Mishchenko, A. V. Shytov, and P. G. Silvestrov, Phys. Rev. Lett. 104, 156806 (2010).

${ }^{10}$ A. Vakil and N. Engheta, Science 332, 1291 (2011).

${ }^{11}$ F. H. L. Koppens, D. E. Chang, and F. J. García de Abajo, Nano Lett. 11, 3370 (2011).

${ }^{12}$ A. Yu. Nikitin, F. Guinea, F. J. García-Vidal, and L. Martín-Moreno, e-print arXiv:1104.3558v1.

${ }^{13}$ L. Brey and H. A. Fertig, Phys. Rev. B 75, 125434 (2007).

${ }^{14}$ P. G. Silvestrov and K. B. Efetov, Phys. Rev. B 77, 155436 (2008).

${ }^{15}$ B. Wunsch, T. Stauber, F. Sols, and F. Guinea, New J. Phys. 8, 318 (2006).

${ }^{16}$ E. H. Hwang and S. Das Sarma, Phys. Rev. B 75, 205418 (2007).

${ }^{17}$ L. A. Falkovsky, Phys. Usp. 51, 887 (2008).

${ }^{18}$ E. H. Hwang et al., Phys. Rev. Lett. 98, 186806 (2007). 